\title{
Reversible P3HT/Oxygen Charge Transfer Complex Identification in Thin Films Exposed to Direct Contact with Water
}

\author{
Sebastiano Bellani, ${ }^{\dagger} \S$ Daniele Fazzi, ${ }^{\S}, \|$ Paola Bruno, ${ }^{\S}$ Ester Giussani, ${ }^{\S}$, Eleonora Valeria Canesi, ${ }^{\S}$ \\ Guglielmo Lanzani, ${ }^{\dagger}, \dagger$ and Maria Rosa Antognazza $*, \S$ \\ ${ }^{\dagger}$ Dipartimento di Fisica, and ${ }^{\ddagger}$ Dipartimento di Chimica, Materiali e Ingegneria Chimica 'Natta', Politecnico di Milano, Piazza L. Da \\ Vinci 32, 20133 Milano, Italy \\ ${ }^{\S}$ Center for Nanoscience and Technology @PoliMi, Istituto Italiano di Tecnologia, via Pascoli 70/3, 20133 Milano, Italy \\ "Max-Planck Institut fur Kohlenforschung, Kaiser-Wilhelm-Platz 1, D-45470 Mülheim an der Ruhr, Germany
}

Received: December 5, 2013

Revised: March 4, 2014

Published: March 5, 2014

\section{INTRODUCTION}

Many emerging technologies in neuroscience, biology, or energy exploit organic semiconductors in contact with a liquid phase, for instance, an electrolyte or a cell culturing medium. Practical demonstrations include biosensors, ${ }^{1,2}$ electrodes, and transistors for stimulation and recording of in vitro neural networks, ${ }^{3-5}$ biomedical devices for optically driven neuronal stimulation and artificial retinal prosthesis, ${ }^{6-10}$ or electrochemical cells for water splitting and hydrogen production. ${ }^{11-14}$ The urgent need for a detailed characterization of the polymer-liquid interface is the natural consequence of such applications and proof-of-concept devices, and it should include the phenomena of contamination, doping, and possible degradation of the organic semiconductor in direct contact with the liquid. In particular, the effect of visible light should be also included in the analysis, not only because it is well-known that photoexcitation enhances doping effects, but also because many of the above-mentioned applications rely on direct exposure to light, as a photoexcitation source or as a natural working condition for in vitro and in vivo application. Traditionally, however, much more is known about contamination in air or in a humid environment with respect to the case of immersion in water. Even though oxygen is the main expected cause of degradation in both situations, it should be noted that surface contamination and diffusion in the polymer films could be very different, due to the different physicalchemical properties and the different electrostatic environment of the interface.

In all mentioned applications, the gold standard organic semiconductor is the alkyl-thiophene-based polymer poly(3hexylthiophene) (rr-P3HT). Extensive characterization of rrP3HT degradation processes upon the effect of UV-vis light and oxygen led to the identification of both irreversible and reversible effects. Irreversible degradation, quantitatively studied by means of different techniques (UV-vis absorption spectroscopy and photoluminescence (PL), ${ }^{15-24}$ infrared (IR) spectroscopy, ${ }^{16,25}$ electron paramagnetic resonance spectrosco$\mathrm{py}^{26} \mathrm{X}$-ray and ultraviolet photoelectron spectroscopy ${ }^{27}$ ), has been attributed to polymer oxidation by radical chain mechanisms, resulting in the destruction of the $\pi$-conjugated system and the formation of covalent bonds between oxygen and carbon, such as carbonyl and carboxyl group. ${ }^{16}$ It has been 
widely reported that irreversible processes are mostly activated by UV-light, while they are strongly reduced upon visible-light excitation.

Reversible effects have been explained by the formation of a complex between the polymer and the oxygen, with a partial redistribution of the electronic charge: ${ }^{18,22-29}$ the charge transfer from $\mathrm{P} 3 \mathrm{HT}$ to oxygen yields a negatively charged oxygen molecule and a mobile hole on the P3HT chain, according to Scheme 1. Electron transfer can take place in the dark, but its rate is considerably speeded up by light exposure.

\section{Scheme 1. P3HT/Oxygen Complex Formation}

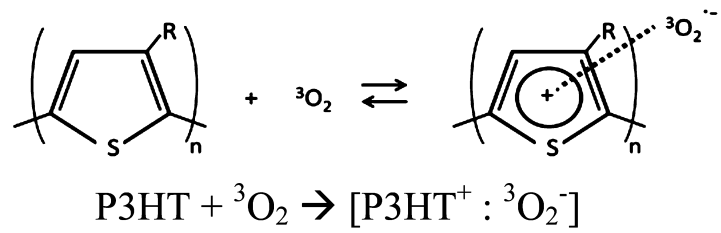

Recently, it was reported ${ }^{25}$ that moisture plays a key-role in the photoactivated reversible oxidation of thiophene-based materials, according to the claim that hydrated $\mathrm{O}_{2}\left(\mathrm{H}_{2} \mathrm{O}\right)_{n}$ clusters have larger electron affinity than the $\mathrm{O}_{2}$ molecule itself. However, it is still not clear ${ }^{15}$ whether water acts solely as a solvent, facilitating the diffusion of reactants, whether it stabilizes polar or ionic intermediates, or whether it takes part in the process itself.

In this work, we focus our attention on effects induced by visible light on rr-P3HT thin films in direct contact with water saline solutions. By combining different techniques (optical absorption, PL, water contact angle measurements, Raman and IR spectroscopy), we demonstrate that rr-P3HT undergoes an oxygen-induced photoactivated doping, with subsequent quenching of photoluminescence and a concomitant reduction of surface hydrophobicity. The comparative characterization reported in this work points out that the direct contact with water or aqueous electrolytes does not add further specific catalytic activity (i.e., attributable to possible different origins), as compared to humid air; conversely, the reduced number of oxygen molecules in water turns out to be a limiting factor to photoactivated doping reactions, counterbalancing the enhancement activity of the hydrated $\mathrm{O}_{2}\left(\mathrm{H}_{2} \mathrm{O}\right)_{n}$ clusters. Raman and IR studies, both experimental and theoretical (Density Functional Theory, DFT modeling), rule out a direct involvement of water molecules in photoactivated doping processes, suggesting instead an active role of the only oxygen. Our data provide an insightful picture of the reversible lightassisted formation of a charge transfer complex between polymer and molecular oxygen and first unambiguously identify its vibrational spectral signatures. This finding turns out to be useful, in addition to the understanding of hybrid, polymer/ electrolytes interfaces, also in the organic photovoltaic field, where the debate on mechanisms leading to degradation is still extremely active. ${ }^{30}$ In perspective, this work suggests that barrier layers developed for devices working in air could be as well efficient in water.

\section{METHODS}

Polymer Thin Films Preparation. rr-P3HT (SigmaAldrich) has a regioregularity of $99.5 \%$ and an average molecular weight of 54000-75000 g/mol; it has been used without any further purification. As thorough cleaning of the substrate was required, rinses were performed in an ultrasonic bath with the following sequential solutions: a specific tensionactive agent in a water solution (HELLMANEX II, 3\%), deionized water, pure acetone, and isopropyl alcohol. Chlorobenzene solutions of rr-P3HT were prepared in a concentration of $15 \mathrm{~g} / \mathrm{L}$ and subsequently heated to $50{ }^{\circ} \mathrm{C}$ for $20 \mathrm{~min}$, stirred, and finally deposited onto previously heated ITO-covered squared glass substrates (18 mm side, $180 \mu \mathrm{m}$ thickness, $10 \Omega /$ sq resistance; Nanocs) by spin-coating. The spinning parameters (first step $800 \mathrm{rpm}$, angular acceleration $1500 \mathrm{rad} / \mathrm{s}^{2}$, rotation duration $30 \mathrm{~s}$; second step $1500 \mathrm{rpm}$, angular acceleration $1000 \mathrm{rad} / \mathrm{s}^{2}$, rotation duration $30 \mathrm{~s}$ ) were carefully selected to obtain suitable optical quality and film thickness $(\sim 100 \mathrm{~nm})$. After deposition, organic layers were annealed for $20 \mathrm{~min}$ at $150{ }^{\circ} \mathrm{C}$, to evaporate residual solvent in the polymer films. The preparation of the solution, the deposition of the $\mathrm{P} 3 \mathrm{HT}$ film, and the annealing process were performed under inert atmosphere.

Because this work is based on exposure of samples to different environmental conditions, we then treated and stored samples by adopting different protocols: a first set was properly stored under controlled nitrogen atmosphere to keep them free of moisture, and extracted from the glovebox chamber just for the time needed for the measurements. For absorption and photoluminescence measurements, this set was encapsulated to avoid contact with air. Part of these samples was stored in dark conditions, and hereinafter they will be indicated by ND (nitrogen + dark); remaining samples were exposed to continuous white light illumination for $24 \mathrm{~h}$, hereinafter indicated by NL (nitrogen + light). A second sample set was exposed after fabrication to open air conditions; one-half of them were subjected to $24 \mathrm{~h}$ of illumination, while the others were kept in the dark (air + light and air + dark, hereinafter AL and $\mathrm{AD}$, respectively). Finally, a third set of samples was placed in direct contact with saline water solutions, some of them in dark conditions (water + dark, WD), some others upon continuous illumination (water + light, WL). The saline solution is obtained by dissolving sodium chloride in Millipore water, 0.2 $\mathrm{M}$ concentration. All reported data for each sample set are mediated over three samples. After measurements, some samples were reannealed in inert atmosphere for $15 \mathrm{~min}$ at $50^{\circ}$ followed by $15 \mathrm{~min}$ at $100^{\circ}$, and then subjected to a second measurement (hereinafter, reannealed samples are indicated by ALR and WLR).

Light and Oxygen Exposure. The whole sample area was illuminated for $24 \mathrm{~h}$ by using a white collimated LED (MCWHL2-C4, Thorlabs). The power density on the sample was $460 \mathrm{~W} / \mathrm{m}^{2}$. For the IR illumination, the source was a tungsten halogen light source (ASB-W-030, Spectral Products), with an approximate power density on the sample of $4 \mathrm{~kW} / \mathrm{m}^{2}$. When spectral filtering was required, we used a combination of cut-on and cutoff optical filters (VPFS-VIS-25.0M, CVI Melles Griot). Some samples were exposed to pure oxygen overpressure ( $1.5 \mathrm{~atm})$ for $24 \mathrm{~h}$, by using a sealed reaction chamber. The same oxygen pressure has been used for samples in contact with water, reaching different steady-state concentration of dissolved oxygen in water (approximately $65 \mathrm{mg} / \mathrm{L}$ ). Finally, some samples were exposed to deoxygenated water in equilibrium with controlled nitrogen atmosphere, resulting in negligible concentration of dissolved oxygen in water.

Absorption and Photoluminescence Spectra. Absorption spectra were recorded by using a spectrophotometer (PERKIN ELMER Lambda 1040) in transmission mode. 

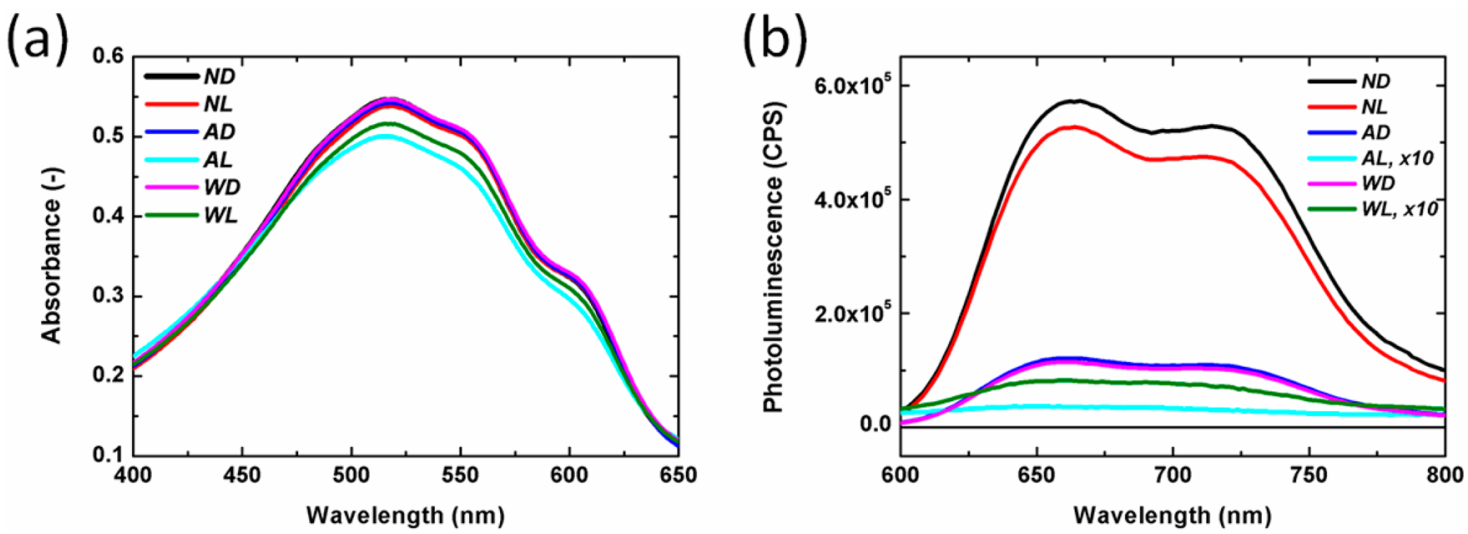

Figure 1. Absorption (a) and photoluminescence (b) spectra of P3HT samples exposed to different environmental conditions: stored in dark conditions and upon controlled atmosphere (nitrogen dark, ND, black line) or upon air (air dark, AD, blue line); illuminated continuously with visible light in nitrogen (nitrogen light, NL, red line) or in air (air light, AL, cyan line); exposed to the direct contact with water in dark (water dark, $\mathrm{WD}$, magenta line) or upon light (water light, WL, green line).

Photoluminescence (PL) spectra were acquired by using a JOBIN-YVON spectrofluorimeter; the excitation wavelength was set at the polymer absorption peak wavelength $(520 \mathrm{~nm})$. Water contact angles were obtained by using a dedicated instrument (DATAPHYSICS, OCA-15) and distilled water drops $(2 \mu \mathrm{L})$ as a reference.

Raman and IR Spectroscopy Measurements. Raman spectra were recorded by using visible (VIS) and near-infrared diode lasers, emitting, respectively, at 532 and $785 \mathrm{~nm}$, and a $\mathrm{Nd}$ :YAG laser for excitation at $1064 \mathrm{~nm}$. For 532 and $785 \mathrm{~nm}$ excitation laser, the microscope was part of Renishaw microscope in via Raman microscope in a back scattering configuration, and of Horiba Jobin Yvon HR800 micro-Raman spectrometer system, both including a monochromator, notch filters system, and a charge coupled detector. Samples were placed on the stage of a Leica microscope, equipped with $5 \times$, $20 \times, 50 \times$, and $100 \times$ short and long working distance objectives. In all measurements, laser power intensity on the sample was kept at values lower than $0.03 \mathrm{~mW}$ to avoid laserinduced sample degradation.

FT-Raman spectra of P3HT films were measured with a Raman Nicolet NXR 9650 spectrometer with MicroStage using a $1064 \mathrm{~nm}$ excitation wavelength (resolution $4 \mathrm{~cm}^{-1}$, number of scans 512, laser power $200 \mathrm{~mW}$ ).

Spectra were typically recorded in the range $600-2000 \mathrm{~cm}^{-1}$ in the Stokes region and were calibrated against the $520.5 \mathrm{~cm}^{-1}$ line of an internal silicon wafer. The signal-to-noise ratio was enhanced by repeated acquisitions. The measurements were conducted at room temperature, and the resulting spectral resolution was $0.4 \mathrm{~cm}^{-1}$.

FT-IR spectra of P3HT films were measured at room temperature using an FT-IR Nicolet Nexus spectrometer with a Thermo Electron Continuum microscope (resolution $4 \mathrm{~cm}^{-1}$, number of scans 256). A double transmission mode was employed for the measurements.

Band fitting was done using a Lorentzian function with the minimum number of components bands used for the fitting process, and the fitting process was undertaken until reproducible results were obtained with squared correlation greater than 0.995 .

Quantum Chemical Calculations. First-principles density functional theory (DFT) calculations were carried out to model the oxygen versus neutral polymer interaction. Grimme dispersion corrected DFT functional B97D ${ }^{31}$ with a triple split Pople basis set with diffusion and polarization functions, 6$311 \mathrm{G}(\mathrm{d}, \mathrm{p})$, was used. Counterpoise basis set superposition error $\left(\mathrm{BSSE}^{32}\right)$ was applied for evaluating the stabilization energy $(\Delta E)$ calculated as the difference between the total energy of the oxygen-polymer complex and the total energies of molecular oxygen and polymer considered as separated (e.g., not interacting units). The oxygen molecule was considered in its stable triplet ground state at the unrestricted UB97D/6$311 \mathrm{G}^{* *}$ level. The polymer was studied by following an oligomeric approach; ${ }^{33}$ in particular, P3HT was modeled by considering six repeat units featuring methyl groups instead of the alkyl chains. The following strategy was adopted for describing the possible intermolecular interactions: (i) isolated P3HT and ${ }^{3} \mathrm{O}_{2}$, (ii) P3HT with one molecular oxygen named P3HT: ${ }^{3} \mathrm{O}_{2}$ complex, and (iii) $\mathrm{P} 3 \mathrm{HT}$ with two molecular oxygen labeled as P3HT:2 $\left({ }_{3} \mathrm{O}_{2}\right)$ complex. The same procedure was applied for modeling the interaction between P3HT and one water molecule. All of the optimized geometries represent a stable minimum without featuring imaginary frequencies. IR and Raman spectra were computed at the same DFT level of theory, and vibrational frequencies were scaled by a factor of 1.02. All calculations were carried out by using the Gaussian 09 program. $^{34}$

\section{EXPERIMENTAL RESULTS}

For all measurements reported below, samples were prepared and stored in different environmental conditions, aiming at discriminating the effect of oxygen, light, and direct contact with water saline solutions (see Methods for protocol details and adopted nomenclature) on polymer properties.

Absorption and Photoluminescence Spectra. Figure 1a shows the absorption spectra for P3HT films exposed to different environmental conditions for $24 \mathrm{~h}$ : ND (black), NL (red), $\mathrm{AD}$ (blue), $\mathrm{AL}$ (cyan), WD (magenta), and WL (olive). The line shape is the same in all samples, showing a broad absorption band peaking at $520 \mathrm{~nm}$, with a secondary peak at $554 \mathrm{~nm}$ and a shoulder at $610 \mathrm{~nm}$. In all samples stored in dark conditions (i.e., $\mathrm{ND}, \mathrm{AD}$, and $\mathrm{WD}$ ), the absorbance intensity does not show significant differences. Conversely, upon visible light irradiation, two important effects can be observed in samples exposed both to air (AL) and to water (WL): a decrease of the overall absorbance, less pronounced in the water-exposed sample (8.4\% in $\mathrm{AL}$ and $5.5 \%$ in $\mathrm{WL}$, evaluated 
in correspondence to the maximum absorption peak as a relative variation with respect to the ND sample), and a slight blue-shift (approximately $10 \mathrm{~nm}$ ). Note that these features are not present in the NL sample. The reannealing of the samples in inert conditions (see Methods for details) does not lead to changes in the reported spectra (see Supporting Information, Figure S1): after exposure to light and open air/water, it is not possible to restore preconditioning absorption characteristics.

Figure $1 \mathrm{~b}$ shows PL spectra for the same series of samples and comparable excitation conditions. PL is heavily affected: in samples exposed to air or water saline solution, it is reduced to about $1 / 6$ of its original value. Samples kept in air or water under light $(\mathrm{AL}, \mathrm{WL})$ show an almost complete suppression of emission. Because, as we discussed above, absorption changes are small, the main cause of PL quenching should be the enhancement of nonradiative decay. We notice that the contact with the liquid does not introduce further effects; rather it seems to reduce the overall PL decrease.

Water Contact Angle (WCA) Measurements. Figure 2 shows the water contact angle (WCA) measurements upon

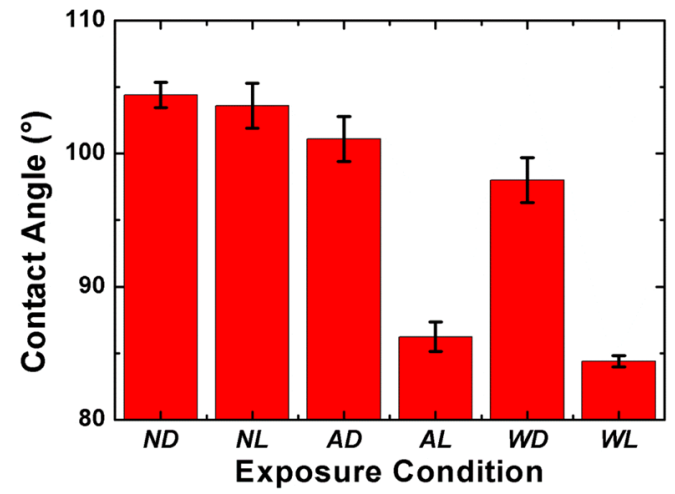

Figure 2. Water contact angle measurements in samples exposed to different conditions (ND, nitrogen, in dark; NL, nitrogen, upon visible light; $\mathrm{AD}$, air, in dark; $\mathrm{AL}$, air, upon visible light; $\mathrm{WD}$, immersed in water, in dark; WL, immersed in water, upon visible light).

exposure to different environmental conditions. ND/NL samples could not be encapsulated in this case: exposure to ambient conditions was therefore kept to a minimum, enough to carry out the measurements, yet fast contamination might have partially affected the outcome. Considering the values for ND or NL (almost identical) as initial condition, the general trend is a reduced hydrophobicity (smaller contact angle) for the exposed samples. In dark, contact with liquid solution leads to a more hydrophilic surface with respect to the contact with air, determining a difference of $3^{\circ}$ in the contact angle of the two samples ( $\mathrm{AD}$ vs $\mathrm{WD})$. The observed variations are larger upon the combined action of open air/water and light. Indeed, upon illumination the contact angles are drastically reduced, with a relative variation with respect to dark conditions of $14.7 \%$ and $13.9 \%$ in $\mathrm{AL}$ and $\mathrm{WL}$ samples, respectively.

Raman and IR Spectroscopy. Figure 3 reports the Raman spectra recorded in resonant conditions (excitation wavelenght: $532 \mathrm{~nm}$ ). We focus in particular on the $1350-1500 \mathrm{~cm}^{-1}$ spectral range (zoomed in Figure $3 \mathrm{~b}$ ), known to be the active Raman region sensitive to the $\pi$-electron delocalization (i.e., the conjugation length) of the P3HT chain. ${ }^{35}$ The two main peaks at 1378 and at $1445 \mathrm{~cm}^{-1}$ have been widely characterized in the literature and have been assigned to $\mathrm{C}-\mathrm{C}$ stretching and in-

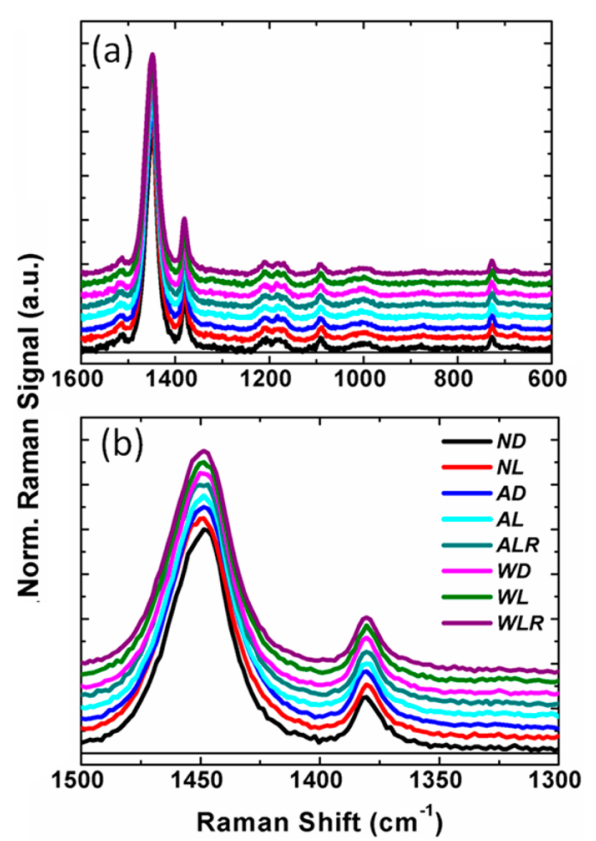

Figure 3. (a) Resonant Raman spectra in samples exposed to different conditions (excitation wavelength: $532 \mathrm{~nm}$ ). (b) Zoom over the spectral range $1300-1500 \mathrm{~cm}^{-1}$, sensitive to $\pi$-electron delocalization of the P3HT chain.

phase $\mathrm{C}=\mathrm{C} / \mathrm{C}-\mathrm{C}$ stretching/shrinking of the aromatic thiophene ring, ${ }^{36,37}$ respectively.

The lack of any significant change in the Resonant Raman spectra for samples exposed to different conditions suggests that structural variations caused by the polymer/environment interactions are not evidenced at this excitation wavelength, although the presence of well-defined peaks around the 1200 and $720 \mathrm{~cm}^{-1}$ spectral region cannot exclude the presence of polaronic-like species in the polymer, as reported by Pron et al. $^{38}$ and Bayley et al. ${ }^{39}$

Figure 4 shows Fourier transform (FT) Raman spectra acquired by using for excitation a near-infrared laser (Nd:YAG laser at $1064 \mathrm{~nm}$ ).

Out-of-resonance condition allows a more accurate analysis of the low-intensity modes. One of the major effects induced by photoirradiation is the enhancement of peaks around 1200 and $720 \mathrm{~cm}^{-1}$ (AL and WL curves), attributed in the literature to the presence of polaronic-like species ${ }^{37-40}$ and in particular to the inter-ring $\mathrm{C}-\mathrm{C}$ stretch mode $\left(1208 \mathrm{~cm}^{-1}\right)$, the $\mathrm{C}-\mathrm{H}$ bending mode with the $\mathrm{C}-\mathrm{C}$ inter-ring stretch mode (1180 $\left.\mathrm{cm}^{-1}\right)$, and the $\mathrm{C}-\mathrm{S}-\mathrm{C}$ deformation mode $\left(728 \mathrm{~cm}^{-1}\right)$.

After reannealing the previously enlightened sample (ALR and WLR curves), these bands are considerably reduced.

Figure $4 \mathrm{~b}$ zooms on the most intense spectral range, 1350$1500 \mathrm{~cm}^{-1}$. Again the main differences among the considered environmental conditions are due to a combined effect of light and air/water ( $\mathrm{AL}$ and $\mathrm{WL}$ samples). Moreover, there is no clear difference between samples exposed to open air and those exposed to a direct contact with water. Upon illumination, in air as well as in liquid, the peak at $1379 \mathrm{~cm}^{-1}$ shows a slight broadening $\left(\sim 2 \mathrm{~cm}^{-1}\right)$, while the peak at $1444 \mathrm{~cm}^{-1}$ shows a very slight red shift (less than $2 \mathrm{~cm}^{-1}$ ). Notably, important variations in terms of broadening between the two main peaks (area around $1420 \mathrm{~cm}^{-1}$ ) are clearly noticeable. In the dark ( $\mathrm{AD}$ and WD samples), the Raman peak at $1443 \mathrm{~cm}^{-1}$ can be fully reproduced by just two Lorentzian functions (solid green 

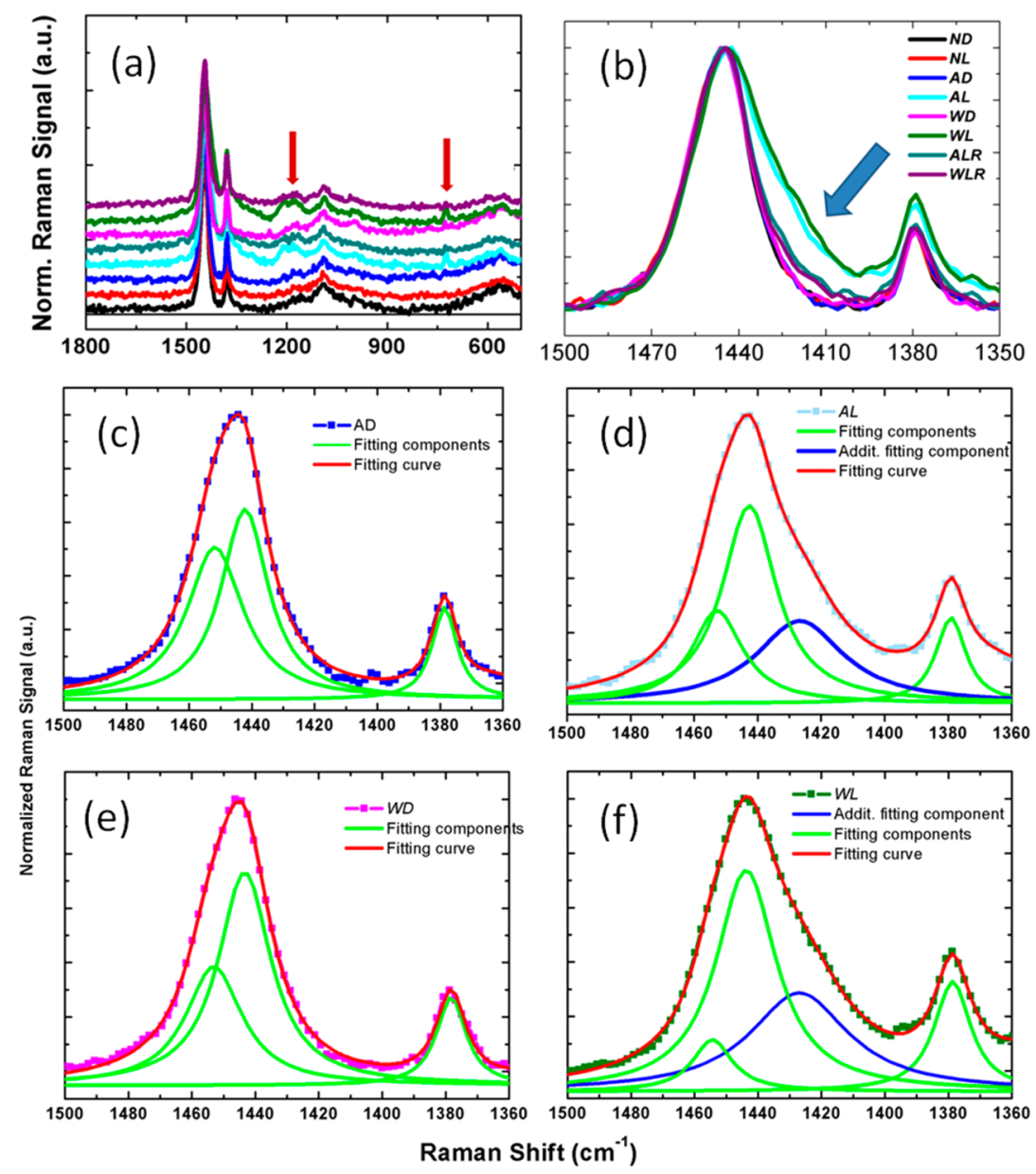

Figure 4. Out-of-resonance FT-Raman spectra (excitation wavelength: $1064 \mathrm{~nm}$ ). (a) The appearance of features round 1200 and $720 \mathrm{~cm}^{-1}$ is visible only in samples exposed both to oxygen (either in water, olive line $\mathrm{WL}$, or in air, cyan line $\mathrm{AL}$ ) and to light. The direct contact with water does not produce additional remarkable effects, as compared to samples exposed to open air. (b) Zoom on the $1500-1350 \mathrm{~cm}^{-1}$ spectral range. The additional contribution of a band arising round $1420 \mathrm{~cm}^{-1}$ is clearly evinced, only in the case of samples exposed to the combined action of light and oxygen (contained in air or in water), AL and WL curves. The additional band disappears upon reannealing the samples (ALR and WLR). (c)-(f) Fitting of AL and WL spectra requires an additional band peaking at $1425 \mathrm{~cm}^{-1}$ (solid blue line in panels (d) and (f)), whose contribution is fully negligible in $\mathrm{AD}$ and $\mathrm{WD}$ samples.

lines in Figure $4 \mathrm{c}$ and $\mathrm{e}$ ), attributed in the literature to the $\mathrm{C}=$ $\mathrm{C}$ aggregated and unaggregated bonds. ${ }^{35,37} \mathrm{In} \mathrm{AL}$ and $\mathrm{WL}$ samples, an additional broad peak around $1425 \mathrm{~cm}^{-1}$ is needed to obtain a proper fitting (see Figure $4 \mathrm{~d}$ and $\mathrm{f}$, solid blue line). Detailed fitting values are reported in the Supporting Information (Table S1). Interestingly, reannealing of the illuminated samples (ALR and WLR) leads to a considerable reduction of the additional band. The relative weight changes, with respect to the intensity of the $\mathrm{C}=\mathrm{C}$ mode used as a reference, $I_{1425} / I_{\mathrm{C}=\mathrm{C}}$, are from $53 \%$ and $62 \%$ (WL and $\mathrm{AL}$, respectively) to $4 \%$ and $8 \%$ (upon further annealing, WLR and ALR).

Similar observations can be derived by careful inspection of preresonant Raman spectra (excitation wavelength at $785 \mathrm{~nm}$ ), reported in the Supporting Information (see experimental spectra and fitting curves in Figure S2, and fitting parameters in Table S2), even though the contribution of the additional band is less evident.

IR spectra acquired in samples exposed to different environmental conditions significantly contribute to complete our study (Figure 5).

Samples exposed to light, both in liquid and in air (WL and $\mathrm{AL})$, show additional bands in the $1100-1350 \mathrm{~cm}^{-1}$ spectral

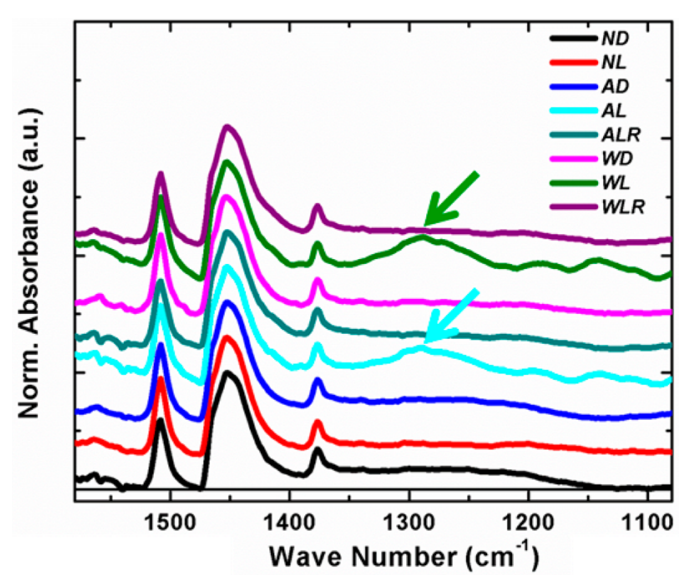

Figure 5. IR spectra of samples exposed to different conditions. Reversible effects in the $1100-1350 \mathrm{~cm}^{-1}$ spectral range are observed in the presence of oxygen and light (AL and WL), and disappear upon reannealing (ALR and WLR).

range with respect to reference samples stored in nitrogen (ND and $\mathrm{NL}$ ) or not exposed to illumination ( $\mathrm{AD}$ and $\mathrm{WD})$. After 

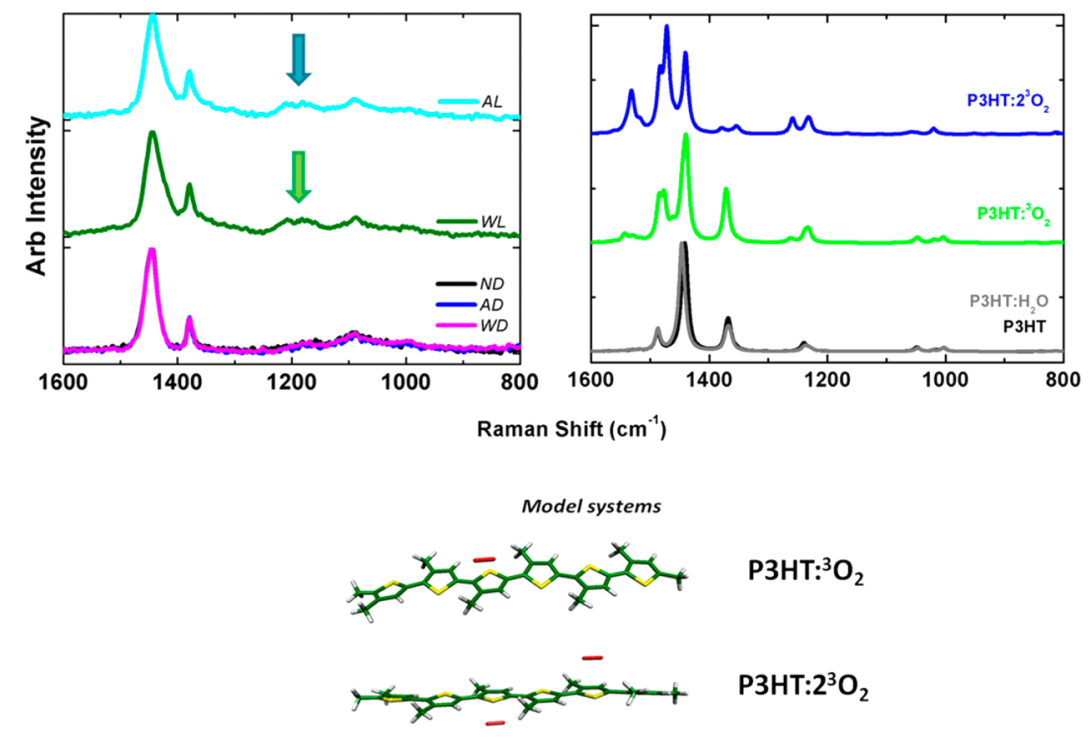

Figure 6. Comparison between experimental (left) and computed B97D/6-311G** (right) Raman spectra for P3HT oligomer, P3HT: $\mathrm{H}_{2} \mathrm{O}$, and P3HT: $n\left({ }^{3} \mathrm{O}_{2}\right)$. Optimized molecular structures for P3HT: $n\left({ }^{3} \mathrm{O}_{2}\right)$ are reported in the bottom of the figure.

reannealing (WLR and ALR), these additional IR bands are removed, proofing the high sensitivity of the IR technique to probe different environments and conditions.

\section{DISCUSSION AND THEORETICAL MODELING}

Three common and relevant features can be summarized from the experimental data: (i) the direct contact with water does not determine specific degradation effects, as compared to what is observed upon contact with open air; (ii) vibrational features observed in Raman, FT-Raman, and IR spectra are associated with a reversible effect, being almost completely suppressed by reannealing the exposed samples; and (iii) the reversible effects are due to the combined action of visible light illumination and contact with air/water that means samples exposed to air or water but kept in the dark do not show specific features, as compared to the encapsulated ones.

$\mathrm{UV}$-vis absorption and PL data in this work are consistent with results previously reported in the literature, and interpreted on the basis of irreversible (i.e., polymer oxidation by radical chain mechanisms and conjugation loss) and reversible (i.e., charge transfer complex formation between polymer and oxygen, accelerated in the presence of light) processes. Our data demonstrate that the direct contact between the polymer films and a liquid saline environment does not add a further component to the degradation processes. Strikingly, it is the other way around: the presence of water reduces part of the degradation processes, possibly due to a lower concentration of oxygen molecules in water as compared to open air or to a different electrostatic coupling with the environment, leading to a different shielding of electric dipoles present at the interface between the polymer and the air/water.

WCA measurements show that doping effects do affect the surface of the polymer at interface. The nonpolar chains in neutral P3HT molecules lead to a hydrophobic surface; ${ }^{41,42}$ indeed, the measured contact angle for bare P3HT (ND sample) is in the order of $105^{\circ}$. Doping processes, attributed to the presence of oxygen, are associated with the presence of excited dipoles that enhance the electrostatic interaction and reduce hydrophobicity; consequently, upon exposure to air and liquid solution, the contact angles systematically decrease ( $\mathrm{AD}$ and WD). Strikingly, upon the combined effect of oxygen and light (AL and WL), the contact angle is drastically reduced, to less than $85^{\circ}$. The relative variation with respect to films kept in the dark is even larger for samples exposed to air as compared to those exposed to liquid solution.

Resonant Raman spectra do not clearly put in evidence irreversible processes (as those observed by UV-vis absorption measurements), meaning that change in the polymer conjugation lengths, upon aerobic conditions, visible light illumination, and direct contact with water, is not a pronounced, substantial effect. Strong oxidation of the material can be also positively excluded by the absence, in all samples sets and at each excitation wavelength used in this study, of the $1624 \mathrm{~cm}^{-1}$ peak attributed to the quinoid structure. ${ }^{40}$

Conversely, upon visible irradiation and oxygen interaction, nonresonant Raman spectra show a clear enhancement of the peaks at 1420,1200 , and $720 \mathrm{~cm}^{-1}$, and the same holds true for IR bands in the spectral region around $1250 \mathrm{~cm}^{-1}$. The contact with saline water does not qualitatively affect the appearance of evidenced spectroscopic features, suggesting the absence of a direct interaction between the polymer and the water molecules. We tentatively assign these features to the formation of a charge transfer complex between P3HT and molecular oxygen ${ }^{3} \mathrm{O}_{2}$ (hereafter reported as P3HT: $n\left({ }^{3} \mathrm{O}_{2}\right)$, with $n$ the number of oxygen molecule per P3HT chain).

To corroborate this hypothesis, we measure FT-Raman and IR spectra in samples exposed to compressed molecular oxygen atmosphere (1.5 atm), both under dark and upon light (Figures S3 and S4 in the Supporting Information). The results confirm that the film exposed to over pressure of oxygen in dark $(A D$, over oxygenated) does not show any difference with respect to encapsulated samples (ND), while upon illumination (AL, over oxygenated) the peaks attributed to the formation of P3HT: $n\left({ }^{3} \mathrm{O}_{2}\right)$ complexes are strongly enhanced. Upon reannealing in nitrogen atmosphere ( $\mathrm{AL}$, over oxygenated after reannealing), the bands are almost completely removed, confirming the reversible nature of the process. The contact with over oxygenated water $(\mathrm{WD}$, over oxygenated and $\mathrm{WL}$, over oxygenated) confirms the picture, enlighting the active role of the oxygen molecules and excluding other possible 


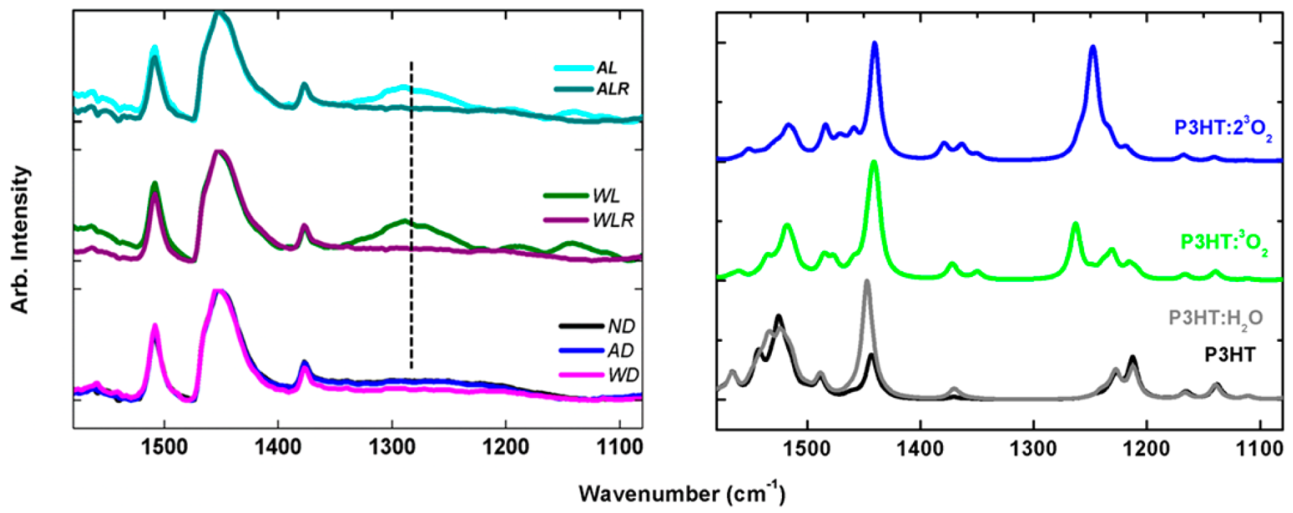

Figure 7. Comparison between experimental (left) and computed B97D/6-311G** (right) FT-IR spectra for P3HT oligomer $\left(\right.$ black), $\mathrm{P} 3 \mathrm{HT}: \mathrm{H}_{2} \mathrm{O}$ (gray), P3HT: ${ }^{3} \mathrm{O}_{2}$ (green), and P3HT: $n\left({ }^{3} \mathrm{O}_{2}\right)$ (blue).

degradation mechanisms. The concentration of oxygen in over oxygenated air and over oxygenated water is 7 times greater than that of air and water at equilibrium with air. Note that the intensity of both FT-Raman and IR features, attributed to the complex formation, varies of approximately the same factor. Finally, in deoxygenated water (WD, deoxygenated and $\mathrm{WL}$, deoxygenated for dark and light conditions, respectively), no additional bands are present, both in dark and upon light, excluding an active role of the water molecules in doping processes. It is worth noting that oxygen concentration in water is several times less as compared to air (approximately 33 times less, in accordance with Henry's law). However, we observed that the intensity of the bands attributed to P3HT: $n\left({ }^{3} \mathrm{O}_{2}\right)$ complexes is of the same magnitude both for air and for water. This finds an explanation by considering that in water the hydration of oxygen molecules is enhanced (thus leading to more efficient doping), in full agreement with results reported by $\mathrm{Ho}$ and co-workers. ${ }^{25}$

The formation of the charge transfer complex between P3HT and triplet oxygen, both in air and in water, is confirmed by DFT calculations. B97D/6-311G** BSSE corrected calculations were performed on the following systems: (a) isolated P3HT oligomer and molecular oxygen in its triplet ground state $\left({ }^{3} \mathrm{O}_{2}\right)$; (b) P3HT: $n\left({ }^{3} \mathrm{O}_{2}\right)$ molecular complexes with $n=1,2$ (i.e., with one or two molecular oxygens); and (c) P3HT: $\mathrm{H}_{2} \mathrm{O}$ molecular complex. Figure 6 shows the comparison between experimental FT-Raman spectra and DFT computed Raman spectra in systems (a) $-(\mathrm{c})$.

A stabilizing interaction for P3HT: $n\left({ }^{3} \mathrm{O}_{2}\right)$ supramolecular complexes (with $n=1$ or 2 ) has been computed to be of the order of $23 \mathrm{kcal} / \mathrm{mol}$ (BSSE corrected energy), that is, stable at room temperature. Molecular oxygen directly interacts with the $\pi$-electron conjugated thiophene rings (see Figure 6), and the computed intermolecular distance is of about 3.0-3.4 $\AA$.

The computed FT-Raman spectra reproduce the main experimental Raman activations in both pristine polymer (samples not exposed to the combined action of oxygen and light; $\mathrm{ND}, \mathrm{WD}$, and $\mathrm{AD}$ samples) and doped films (AL and $\mathrm{WL}$ samples), in particular in the $1100-1450 \mathrm{~cm}^{-1}$ spectral region (as discussed below). Because of the model systems considered (e.g., finite oligomer length and methyl groups instead of alkyl chains), spurious Raman activations are computed in the 1500 $\mathrm{cm}^{-1}$ region. However, those activations do not affect the spectroscopic assignments related to the vibrational markers we considered in this study (e.g., $1200-1250$ and $800-700 \mathrm{~cm}^{-1}$ ).
One of the major vibrational fingerprints of the molecular oxygen complexes P3HT: $n\left({ }^{3} \mathrm{O}_{2}\right)$ are the peaks around 1200$1250 \mathrm{~cm}^{-1}$ (see also Supporting Information, Figure S6), which are related to the symmetric $\mathrm{O}_{2}$ bond stretching coupled with the P3HT C-C stretching vibrations. An enhancement of the Raman activity for normal modes in the $800-700 \mathrm{~cm}^{-1}$ region is also predicted by increasing the number of interacting oxygen molecules, as reported in the computed spectra (see Figure S7 in the Supporting Information), thus further strengthening the evidence of the formation of a complex between P3HT and triplet oxygen.

Interestingly, no relevant changes in the Raman spectra have been computed between the isolated P3HT chain and the P3HT: $\mathrm{H}_{2} \mathrm{O}$ complex (see Figures S6 and S7 in the Supporting Information), confirming the experimental observation that the direct contact with water does not lead to specific doping-like mechanisms.

These observations are further corroborated by the computed DFT IR spectra (Figure 7) where the bands attributed to the P3HT: $n\left({ }^{3} \mathrm{O}_{2}\right)$ complexes (at 1300-1200 $\mathrm{cm}^{-1}$ ) increase in intensity, in excellent agreement with experiments.

In particular, the interaction between $\mathrm{P} 3 \mathrm{HT}$ and molecular oxygen results in a new IR band peaked at $1250 \mathrm{~cm}^{-1}$. This IR active band is related to the oxygen bond stretching mode (intrinsically IR inactive) that becomes IR active due to the interaction with the P3HT chain (i.e., break of the $\mathrm{O}_{2} D_{\infty h}$ symmetry point group to a lower one).

Furthermore, the higher is the number of oxygen molecules interacting with the polymer $(n=1,2)$, the higher is the IR intensity associated with the $1250 \mathrm{~cm}^{-1}$ band (see theoretical green and blue spectra in Figure 7 and experimental spectra in Supporting Information Figure S4).

On this basis, we analyzed also the computed localized charges (i.e., Mulliken charges) on the oligothiophene and on the molecular oxygen, respectively, resulting in partial charges such as $+0.33 e$ and $-0.33 e$ for $\mathrm{P} 3 \mathrm{HT}:{ }^{3} \mathrm{O}_{2}$, and $+0.54 e$ and $-0.27 e /-0.27 e$ for P3HT: $2\left({ }^{3} \mathrm{O}_{2}\right)$ cases. This behavior resembles the formation of the charge transfer molecular complex between the P3HT and the oxygen, with a positive partial charge localized on the polymer backbone. The observed enhancement of the Raman peaks and IR bands upon oxygen interaction, highly similar (in trend) to the formation of polaronic species on the $\mathrm{P} 3 \mathrm{HT},{ }^{38}$ is thus to be connected to the formation of the charge transfer complex, bringing a positive 
partial charge on the polymer and mimicking consequently the polaron-like optical response.

Our results suggest the possibility of using this band to directly identify the formation of the charge transfer complex between P3HT and oxygen, and, possibly, by carefully controlling the oxygen exposure, to quantitatively assess the doping level in the sample.

Measurements in dependence on bandwidth of photoexcitation (Supporting Information Figure S5) definitely confirm that the identified complex is due to the combined action of both oxygen and visible light: data show that photoexcitation in the same range of polymer optical band gap is a required condition to generate the complex. The complex formation versus excitation wavelength shows saturation below the optical absorption maximum of the polymer. This could be due to an activation barrier for the formation of the P3HT: $n\left({ }^{3} \mathrm{O}_{2}\right)$ complex, corresponding to a wavelength shorter than $650 \mathrm{~nm}$.

\section{CONCLUDING REMARKS}

We extensively characterize the effect of saline aqueous solutions and visible light in thin films of P3HT, a workhorse polymer for photovoltaics, electrochemical devices, and bioelectronic applications. Water, moisture, and oxygen are considered as killers for organic electronic devices. We demonstrate that contact with saline liquid solution is not worse than contact with open air: in both situations the reversible formation of a charge transfer complex between $\mathrm{P} 3 \mathrm{HT}$ and oxygen is the main phenomenon. This is substantially accelerated in the presence of resonantly absorbed light.

In some cases, direct contact with water turns out even to reduce the effect, possibly due to a decreased content of oxygen in water with respect to air, and to a different electrostatic interaction with the environment. In the complex, the oxygen moiety is within a van der Waals radius from the polymer chain and promotes weak charge transfer reactions, resulting in local electrical dipoles. This effect explains the reduced hydrophobicity as due to a different local dielectric environment. The charge transfer complex may also be involved into the nonradiative deactivation of the singlet excited state in the polymer, leading to PL quenching. By combining DFT calculations and experiments, the supramolecular P3HT: $n\left({ }^{3} \mathrm{O}_{2}\right)$ complex is here unambiguously identified by characteristic FT-Raman and IR bands, excluding contributions of different origins. The complex is a precursor site for photoactivated doping: this is detrimental for light emission, but it is not crucial for other applications, in particular for those requiring direct contact with electrolytic media, such as bioorganic interfaces and electrochemical devices for water splitting. In summary, our work provides a detailed characterization of polymer properties in contact with water, validating use of semiconducting polymers in contact with electrolytes and thus paving the way to new, rapidly emerging trends in organic electronics.

\section{ASSOCIATED CONTENT}

Figures S1-S7, Tables S1,S2. This material is available free of charge via Internet.

\section{AUTHOR INFORMATION}

\section{Corresponding Authors}

*E-mail: guglielmo.lanzani@iit.it.

*E-mail: mariarosa.antognazza@iit.it.

\section{Author Contributions}

S.B. performed all experimental measurements, with help from E.G., P.B., and E.C. D.F. performed DFT calculations. G.L. and M.R.A. planned the work and supervised it. S.B. and M.R.A. wrote the manuscript, with contributions from all authors.

\section{Notes}

The authors declare no competing financial interest.

\section{ACKNOWLEDGMENTS}

This work was supported by EU project PHOCS, ENERGY 2012-10.2.1, Future Emerging Technologies Collaborative Project no. 309223; by EU project OLIMPIA, FP7-PEOPLE212-ITN 316832; and by national grant Telethon - Italy, Grant no. GGP12033.

\section{REFERENCES}

(1) Rivnay, J.; Owens, R; Malliaras, G. G. The Rise of Organic Bioelectronics. Chem. Mater. 2013, 26, 679-685.

(2) Muskovich, M.; Bettinger, C. J. Biomaterials- Based Electronics: Polymers and Interfaces for Biology and Medicine. Adv. Healthcare Mater. 2012, 1, 248-266.

(3) Khodagholy, D.; Doublet, T.; Quilichini, P.; Gurfinkel, M.; Leleux, P.; Ghestem, A.; Ismailova, E.; Hervé, T.; Sanaur, S.; Bernard, C.; et al. In Vivo Recordings of Brain Activity Using Organic Transistors. Nat. Commun. 2013, 4, 1575-1582.

(4) Cramer, T.; Chelli, B.; Murgia, M.; Barbalinardo, M.; Bystrenova, E.; de Leeuw, D. M.; Biscarini, F. Organic Ultra-Thin Film Transistors with a Liquid Gate for Extracellular Stimulation and Recordings of Electric Activity of Stem Cell-Derived Neural Network. Phys. Chem. Chem. Phys. 2013, 15, 3897-3905.

(5) Benfenati, V.; Toffanin, S.; Bonetti, S.; Turatti, G.; Pistone, A.; Chiappalone, M.; Sagnella, A.; Stefani, A.; Generali, G.; Ruani, G.; et al. A Transparent Organics Transistor Structure for Bidirectional Stimulation and Recording of Primary Neurons. Nat. Mater. 2013, 12, $672-680$.

(6) Ghezzi, D.; Antognazza, M. R.; Dal Maschio, M.; Lanzarini, E.; Benfenati, F.; Lanzani, G. A Hybrid Bioorganic Interface for Neural Photoactivation. Nat. Commun. 2011, 2, 166-172.

(7) Ghezzi, D.; Antognazza, M. R.; Maccarone, R.; Bellani, S.; Lanzarini, E.; Martino, N.; Mete, M.; Pertile, G.; Bisti, S.; Lanzani, G.; Benfenati, F. A Polymer Optoelectronic Interface Restores Light Sensitivity in Blind Rat Retinas. Nat. Photonics 2013, 7, 400-406.

(8) Martino, N.; Ghezzi, D.; Benfenati, F.; Lanzani, G.; Antognazza, M. R. Organic Semiconductors for Artificial Vision. J. Mater. Chem. B 2013, 1, 3768-3780.

(9) Gautam, V.; Bag, M.; Narayan, K. S. Single-Pixel, Single-Layer Polymer Device as a Tricolor Sensor with Signal Mimicking Natural Photoreceptors. J. Am. Chem. Soc. 2011, 133, 17942-17949.

(10) Antognazza, M. R.; Ghezzi, D.; Musitelli, D.; Garbugli, M.; Lanzani, G. A Hybrid Solid-Liquid Polymer Photodiode for the Environment. Appl. Phys. Lett. 2009, 94, 243501-243503.

(11) Lanzarini, E.; Antognazza, M. R.; Biso, M.; Ansaldo, A.; Laudato, L.; Bruno, P.; Metrangolo, P.; Resnati, G.; Ricci, D.; Lanzani, G. Polymer-Based Photocatalytic Hydrogen Generation. J. Phys. Chem. C 2012, 116, 10944-10949.

(12) Suppes, G.; Ballard, E.; Holdcroft, S. Aqueous Photocathode Activity of Regioregular Poly(3-hexylthiophene). Polym. Chem. 2013, 4, 5345-5350.

(13) Winther-Jensen, B.; Fraser, K.; Ong, C.; Forsyth, M.; Mac Farlane, D. R. Conducting Polymer Composite Materials for Hydrogen Generation. Adv. Mater. 2010, 22, 1-4. 
(14) Winther-Jensen, O.; Winther-Jensen, B.; Mac Farlane, D. R. Photostimulated Electrocatalysis of Water Oxidation by Conjugated Polymers. Electrochem. Commun. 2011, 13, 307-309.

(15) Hintz, H.; Sessler, C.; Peisert, H.; Egelhaaf, H.-J.; Chassé, T. Wavelength-Dependent Pathways of Poly-3-Hexylthiophene PhotoOxidation. Chem. Mater. 2012, 24, 2739-2743.

(16) Manceau, M.; Rivaton, A.; Gardette, J.-L.; Guillerez, S.; Lemaitre, N. The Mechanism of Photo- and Thermooxidation of Poly(3-hexylthiophene) (Р3HT) Reconsidered. Polym. Degrad. Stab. 2009, 94, 898-907.

(17) Abdou, M. S. A.; Holdcroft, S. Mechanism Of Photodegradation of Poly(3-alkylthiophenes) in Solution. Macromolecules 1993, 26, 2954-2962.

(18) Abdou, M. S. A.; Orfino, F. P.; Son, Y.; Holdcroft, S. Interaction of Oxygen with Conjugated Polymers: Charge Transfer Complex Formation with Poly(3-alkylthiophenes). J. Am. Chem. Soc. 1997, 119, $4518-1524$.

(19) Hintz, H.; Egelhaaf, H.-J.; Lüer, L.; Hauch, J.; Peisert, H.; Chassé, T. Photodegradation of P3HT - A Systematic Study of Environmental Factors. Chem. Mater. 2011, 23, 145-154.

(20) Lüer, L.; Egelhaaf, H.; Oelkrug, D.; Cerullo, G.; Lanzani, G.; Huisman, B.; de Leeuw, D. Oxygen-Induced Quenching of Photoexcited States in Polythiophene Films. Org. Electron. 2004, 5, 83-89.

(21) Manceau, M.; Rivaton, A.; Gardette, J.-L. Involvement of Singlet Oxygen in the Solid-State Photochemistry of P3HT. Macromol. Rapid Commun. 2008, 29, 1823-1827.

(22) Seemann, A.; Sauermann, T.; Lungenschmied, C.; Armbruster, O.; Bauer, S.; Egelhaaf, H.-J.; Hauch, J. Reversible and Irreversible Degradation of Organics Solar Cell Performance by Oxygen. Sol. Energy 2011, 85, 1238-1249.

(23) Hintz, H.; Egelhaaf, H.; Peisert, H.; Chassé, T. Photo-Oxidation and Ozonization of Poly(3-hexylthiophene) Thin Films as Studied by UV/VIS and Photoelectron Spectroscopy. Polym. Degrad. Stab. 2010, 95, 818-825.

(24) Hoshino, S.; Yoshida, M.; Uemura, S.; Kodzasa, T.; Takada, N.; Kamata, T.; Yase, K. J. Influence of Moisture on Device Characteristic of Polithiophene-Based Field-Effect Transistor. Appl. Phys. 2004, 95, 5088-5093.

(25) Zhuo, J.-M.; Zhao, L.-H-.; Png, R.-Q.; Wong, L.-W.; Chia, P.-J.; Tang, J.-C.; Sivaramakrishnan, S.; Zhou, M.; Ou, E. C.-W.; Chua, S.-J.; et al. Direct Spectroscopic Evidence for a Photodoping Mechanism in Polithiophene and Poly(Bithiothene-Alt-Thienothiophene) Organic Semiconductor Thin Films Involving Oxygen and Sorbed Moisture. Adv. Mater. 2009, 21, 4747-4752.

(26) Sperlich, A.; Kraus, H.; Deibel, C.; Blok, H.; Schmidt, J.; Dyakonov, V. Reversible and Irreversible Interactions of Poly(3hexylthiophene) with Oxygen Studied by Spin-Sensitive Methods. J. Phys. Chem. B 2011, 115, 13513-13518.

(27) Aygül, U.; Hintz, H.; Egelhaaf, H.-J.; Distler, A.; Abb, S.; Peisert, H.; Chassé, T. Energy Level Alignment of a P3HT/Fullerene Blend during the Initial Steps of Degradation. J. Phys. Chem. C 2013, 117, 4992-4998.

(28) Reese, M . O.; Nardes, A. M.; Rupert, B. L.; Larsen, R. E.; Olson, D. C.; Lloyd, M. T.; Shaheen, S. E.; Ginley, D. S.; Rumbles, G.; Kopidakis, N. Photoinduced Degradation of Polymer and PolymerFullerene Active Layers: Experiment and Theory. Adv. Funct. Mater. 2010, 20, 3476-3483.

(29) Liao, H.-H.; Yang, C.-M.; Liu, C.-C.; Horng, S. F.; Meng, H.-F.; Shy, J.-T. Dynamics and Reversibility of Oxygen Doping and DeDoping for Conjugated Polymer. J. Appl. Phys. 2008, 103, 104506104508 .

(30) Nikiforov, M. P.; Strzalka, J.; Darling, S. B. Delineation of the Effect of Water andOxygen on the Degradation of Organic Photovoltaic Devices. Sol. Energy Mater. Sol. Cells 2013, 110, 36-42.

(31) Grimme, S. Semiempirical GGA-Type Density Functional Constructed with a Long-Range Dispersion Correction. J. Comput. Chem. 2006, 27, 1787-99.
(32) Boys, S. F.; Bernardi, F. The Calculation of Small Molecular Interactions by the Differences of Separate Total Energies. Some Procedures with Reduced Errors. Mol. Phys. 1970, 19, 553-566.

(33) Milani, A.; Brambilla, L.; Del Zoppo, M.; Zerbi, G. Raman Dispersion and Intermolecular Interactions in Unsubstituted Thiophene Oligomers. J. Phys. Chem. B 2007, 111, 1271-1276.

(34) Frisch, M. J.; et al. Gaussian 09, revision B.01; Gaussian, Inc.: Wallingford, CT, 2009.

(35) Gao, Y.; Grey, J. K. Resonance Chemical Imaging of Polythiophene/Fullerene Photovoltaic Thin Films: Mapping Morphology-Dependent Aggregated and Unaggregated $\mathrm{C}=\mathrm{C}$ Species. J. Am. Chem. Soc. 2009, 131, 9654-9662.

(36) Gao, Y.; Martin, T. P.; Niles, E. T.; Wise, A. J.; Thomas, A. K.; Grey, J. K. Understanding Morphology-Dependent Polymer Aggregation Properties and Photocurrent Generation in Polythiophene/ Fullerene Solar Cells of Variable Compositions. J. Phys. Chem. C 2010, 114, 15121-15128.

(37) Tsoi, W. C.; James, D. T.; Kim, J. S.; Nicholson, P. G.; Murphy, C. E.; Bradley, D. D. C.; Nelson, J.; Kim, J.-S. The Nature of In-Plane Skeleton Raman Modes of P3HT and Their Correlation to the Degree of Molecular Order in P3HT:PCBM Blend Thin Films. J. Am. Chem. Soc. 2011, 133, 9834-9843.

(38) Trznadel, M.; Zagórska, M.; Lapkowski, M.; Louarn, G.; Lefrant, S.; Pron, A. UV-VIS-NIR and Raman Spectroelectrochemistry of Regioregular Poly(3-Octylthiophene): Comparison with its NonRegioregular Analogue. J. Chem. Soc., Faraday Trans. 1996, 92, 13871393.

(39) Bayley, P. M.; Winther-Jensen, B.; MacFarlane, D. R.; Rocher, N. M.; Forsyth, M. Enhanced Properties in Chemically Polymerized Poly(Terthiophene) Using Vapour Phase Techniques. React. Funct. Polym. 2008, 68, 1119-1126.

(40) Baibarac, M.; Lapkowski, M.; Pron, A.; Lefrant, S.; Baltog, I. SERS Spectra of Poly(3-Hexylthiophene) in Oxidized and Unoxidized States. J. Raman Spectrosc. 1998, 29, 825-832.

(41) Robinson, L.; Hentzell, A.; Robinson, N. D.; Isaksson, J.; Berggren, M. Electrochemical Wettability Switches Gate Aqueous Liquids in Microfluidic Systems. Lab Chip 2006, 6, 1277-1278.

(42) Robinson, L.; Isaksson, J.; Robinson, N. D.; Berggren, M. Electrochemical Control of Surface Wettability of Poly(3-Alkylthiophenes). Surf. Sci. 2006, 600, L148-L152. 